\title{
MÉTODOS DE INJEÇÃO DA AMOSTRA EM MICROSSISTEMAS ELETROFORÉTICOS
}

\author{
Kemilly M. P. Pinheiro ${ }^{a}$, Kariolanda C. A. Rezende ${ }^{a}$ e Wendell K. T. Coltro ${ }^{\mathrm{a}, \mathrm{b}, *,(\mathbb{C}}$ \\ anstituto de Química, Universidade Federal de Goiás, 74690-900 Goiânia - GO, Brasil \\ 'Instituto Nacional de Ciência e Tecnologia de Bioanalítica, 13083-861 Campinas - SP, Brasil
}

Recebido em 18/08/2020; aceito em 01/12/2020; publicado na web em 20/01/2021

\begin{abstract}
SAMPLE INJECTION METHODS IN ELECTROPHORETIC MICROSYSTEMS. Electrophoresis is by far the most popular separation method implemented in microscale, most probably due to its instrumental simplicity, low cost and portability. Due to the increasing use of miniaturized electrophoretic systems, the study of fundamental aspects can improve the development of methodologies for several applications. One of the major challenges related to electrophoresis chips refers to the sample injection mode, in this way, this study presents a review on sample injection methods for microchip electrophoresis covering electrokinetic and hydrodynamic approaches, describing theoretic and instrumental aspects. Since the sample volume affects the analytical performance, the precise and reproducible control of the sample amount to be introduced into the separation channel is highly desirable to ensure reliable chemical analysis. Electrokinetic modes based on floating, pinched and gated protocols are presented and discussed providing an overview about the electrokinetic control of the sample through three methodologies. In the same way, hydrodynamic techniques including the use of microfabricated valves and pumps, syringe pumps, electronic micropipettes, rubber suction bulb and acupuncture needle are approached, thus expanding the view of hydrodynamic injectors based on classical and alternative methods.
\end{abstract}

Keywords: electrokinetic injection; hydrodynamic injection; microchip electrophoresis; microfluidics.

\section{INTRODUÇÃO}

A miniaturização dos sistemas analíticos é uma das tendências instrumentais que está em constante expansão no âmbito nacional e internacional. ${ }^{1-6}$ A redução da escala convencional para plataformas microfabricadas proporcionou inúmeras vantagens associadas ao consumo de amostra, tempo de análise, portabilidade, geração de resíduos e possibilidade de integração de múltiplas etapas analíticas em um substrato. ${ }^{7-13}$ Em geral, os microssistemas analíticos contêm canais fabricados em substratos planares definidos com largura e profundidade da ordem de dezenas de micrometros. ${ }^{14,15} \mathrm{O}$ transporte de fluidos nos microcanais pode ser controlado por ação de forças eletrocinéticas, hidrodinâmicas, centrífugas ou mesmo pelo fenômeno da capilaridade. ${ }^{16-18}$ A manipulação e o comportamento de fluidos em canais definidos com dimensões micrométricas compreendem a definição do conceito de microfluídica. ${ }^{19,20}$

No Brasil, a microfluídica foi abordada, de forma pioneira, no início dos anos 2000 através da idealização e execução do projeto Multiusuário de Microfabricação (MUSA), organizado pelo Laboratório Nacional de Luz Síncrotron (LNLS), ${ }^{21}$ que integra o Centro Nacional de Pesquisa em Energia e Materiais (CNPEM). Atualmente, o desenvolvimento de sistemas microfluídicos é explorado por grupos de pesquisa situados em, praticamente, todas as regiões brasileiras. A abrangência nacional se deve à contribuição do Laboratório de Microfabricação do CNPEM que, através dos projetos multiusuários e das facilidades instrumentais, viabilizou projetos coordenados por pesquisadores de diferentes regiões.

Devido às vantagens inerentes da miniaturização, o uso de plataformas microfluídicas tem aplicabilidade em diferentes áreas do conhecimento, sendo considerada uma ferramenta inter e multidisciplinar. ${ }^{5,22-29}$ Conforme pesquisa realizada no banco de dados da Web of Science utilizando-se a palavra-chave microfluidics como tópico, foram encontradas aproximadamente 25.000 publicações científicas distribuídas em diversas áreas, como pode ser observado

*e-mail: wendell@ufg.br na Figura 1. Do total de publicações, uma fração representativa indica a predominância nas áreas da nanociência e nanotecnologia, química analítica, instrumentação, química multidisciplinar, engenharia elétrica, métodos bioquímicos, ciência de materiais e física aplicada (Figura 1A). Adicionalmente, a mesma pesquisa foi realizada aplicando-se um filtro para as publicações envolvendo autoria de pesquisadores brasileiros. Ao todo, foram encontradas aproximadamente 170 publicações, com maior destaque para os artigos na área da química analítica (30\% do total) (Figura 1B).

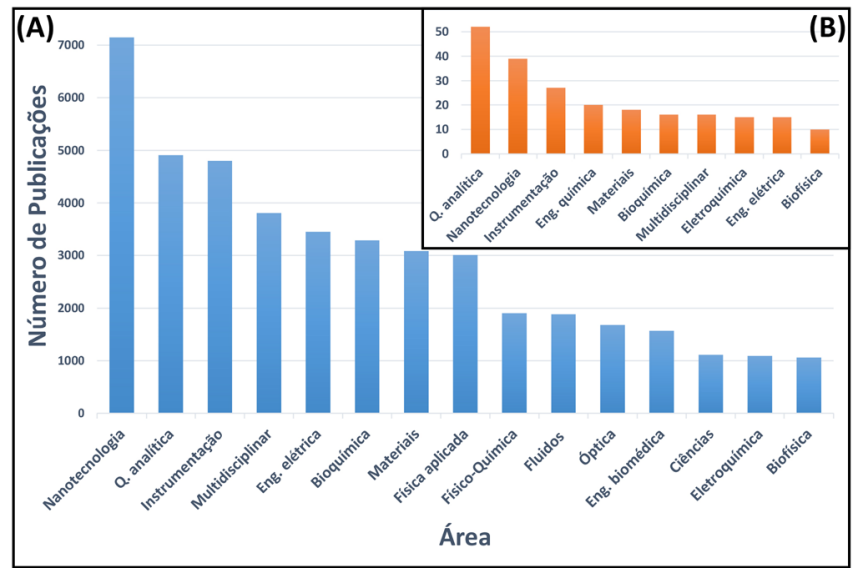

Figura 1. Distribuição do número de publicações envolvendo microfluídica nas principais áreas correlatas considerando (A) o quantitativo total extraído da Web of Science bem como (B) o quantitativo com autoria de pesquisadores brasileiros. Dados extraídos do banco de dados da "Web of Science", em 30/07/2020, usando "microfluidics" como palavra-chave de busca

No âmbito da química analítica, diferentes técnicas podem ser implementadas nas plataformas microfluídicas, incluindo métodos de separação, ${ }^{30-32}$ métodos de preparo de amostra, ${ }^{33-36}$ sistemas de injeção em fluxo, ${ }^{37,38}$ sensores químicos e eletroquímicos, ${ }^{39-43}$ dentre outros. Com relação aos métodos de separação, a eletroforese é a principal 
técnica de eletromigração ${ }^{44-46}$ implementada em microescala. Isso se justifica pela sua simplicidade instrumental quando comparada aos métodos cromatográficos, por exemplo. ${ }^{47}$ Basicamente, os microssistemas eletroforéticos (MSE) requerem, além da plataforma microfluídica, uma fonte de alta tensão e um detector, os quais são utilizados para realizar e monitorar separações analíticas em microcanais, ao invés do uso de capilares ou géis.

A fonte de alta tensão pode ser adaptada dos sistemas eletroforéticos convencionais. No entanto, já existem módulos eletrônicos portáteis que permitem a aplicação de potenciais da ordem de $\pm 1-8 \mathrm{kV}{ }^{48,49} \mathrm{O}$ potencial elétrico é aplicado especialmente para gerar um campo elétrico e promover a migração e separação eletroforética das espécies eletricamente carregadas ou neutras no interior de microcanais. No entanto, o controle eletrocinético é frequentemente utilizado para manipular uma zona de amostragem e permitir a introdução de volumes da ordem de $\mathrm{pL}$ no interior dos canais de separação. ${ }^{6,50}$ Da mesma forma, muitos detectores utilizados nos sistemas convencionais de eletroforese capilar (EC) podem ser acoplados com os MSE, incluindo principalmente a fluorescência, ${ }^{51-53}$ a espectrometria de massas ${ }^{54-56}$ e os métodos eletroquímicos. ${ }^{57-60}$

Em geral, os MSE podem ser fabricados em diferentes substratos, incluindo materiais convencionais e alternativos. Dentre os materiais considerados convencionais, cita-se o vidro, o quartzo e o silício. Nestes substratos, a fabricação de microcanais é normalmente conduzida através de técnicas fotolitográficas, que requerem instrumentação sofisticada e de alto custo, além de salas limpas, o que acaba dificultando a implementação em centros emergentes ou carentes de investimento. ${ }^{4}$ Nos últimos anos, diferentes grupos de pesquisa desenvolveram materiais alternativos, e economicamente acessíveis a todos os níveis de orçamento, para a fabricação de dispositivos microfluídicos. Dentre as plataformas alternativas, pode-se mencionar os polímeros elastoméricos, como o poli(dimetilsiloxano) (PDMS) ${ }^{61,62}$ e a poli(uretana) (PU), ${ }^{63}$ polímeros termofixos, como o polímero à base de tioleno não-estequiométrico (OSTE),${ }^{64}$ e materiais termoplásticos, como o poli(metil metacrilato) (PMMA), ${ }^{65,66}$ o poli(carbonato) (PC) ${ }^{67}$ e o copolímero de olefina cíclica (COC). ${ }^{68}$ Além desses materiais, plataformas compostas de poliéster, ${ }^{69}$ poliéster-toner (PT) ${ }^{70,71}$ papel $^{72,73}$ e, até mesmo, fios de tecido ${ }^{74}$ têm surgido como plataformas promissoras para o desenvolvimento de MSE. Detalhes sobre as principais tecnologias de microfabricação podem ser encontrados em artigos de revisão recentemente publicados. ${ }^{4,6,50}$

A implementação da eletroforese em microssistemas analíticos é desafiadora, pois requer conhecimento instrumental sobre os procedimentos adotados para introdução da amostra, controle eletrocinético e acoplamento com sistemas de detecção. Em cada uma das etapas mencionadas existem várias particularidades que são consideradas desafiadoras. No entanto, quando devidamente compreendidas, podem resultar em sistemas robustos com potencialidade para serem explorados em aplicações diversas, contemplando diferentes ramos da ciência. Dessa forma, esta revisão tem como objetivo apresentar uma abordagem instrumental com relação à uma das principais etapas necessárias nos MSE: a injeção da amostra.

\section{MODOS DE INJEÇÃO DE AMOSTRA}

A miniaturização dos sistemas eletroforéticos apresenta como um dos seus grandes desafios o controle da quantidade de amostra injetada, uma vez que esse afeta diretamente a reprodutibilidade e, consequentemente, a confiabilidade das separações nos MSE. ${ }^{75}$ Assim como nos sistemas eletroforéticos convencionais, ${ }^{44}$ a introdução da amostra nos MSE pode ser realizada através de processos eletrocinéticos ou hidrodinâmicos. ${ }^{16}$ Os dois modos de introdução da amostra apresentam peculiaridades, as quais serão discutidas separadamente.

\section{Injeção eletrocinética}

A injeção eletrocinética tem como principal vantagem a sua praticidade instrumental. Nesse tipo de abordagem, a fonte de alta tensão utilizada para aplicação do potencial elétrico, necessário para a separação eletroforética dos analitos, pode ser também usada para promover a injeção da amostra. Deste modo, o controle dos potenciais elétricos é fundamental para a definição do volume de amostra injetado. ${ }^{16,76}$

Esse tipo de injeção é favorecido quando o fluxo eletrosmótico (FEO) possui a mesma direção da migração dos analitos. Em geral, considerando um sistema convencional de eletroforese capilar, por exemplo, os analitos com mesma carga do potencial aplicado migram no canal em direção ao detector e a quantidade injetada pode ser calculada pela seguinte equação: ${ }^{77}$

$$
Q=\frac{\left(\mu_{e}+\mu_{F E O}\right) \pi r^{2} V t_{i} C}{L}
$$

sendo, $\mu_{e}$ a mobilidade eletroforética, $\mu_{F E O}$ a mobilidade do fluxo eletrosmótico, $r$ o raio do capilar, $V$ a voltagem aplicada, $t_{i} \mathrm{o}$ tempo de injeção, $C$ a concentração do analito e $\mathrm{L}$ o comprimento total do capilar. Desse modo, a quantidade de amostra injetada depende da mobilidade eletroforética de cada analito. Nos sistemas miniaturizados, a quantidade de amostra introduzida no interior dos microcanais não pode ser calculada de maneira análoga aos capilares. Isso ocorre devido às diferenças geométricas bem como no controle dos potenciais aplicados. Além disso, a introdução da amostra pode ser feita baseando-se na difusão da amostra. Nos MSE, três modos eletrocinéticos, denominados como floating, pinched e gated, podem ser utilizados para promover a injeção da amostra. Cada um deles requer definição prévia da geometria do MSE bem como do número de canais disponíveis para aplicação de potenciais elétricos independentes na fonte de alta tensão. ${ }^{78}$

\section{Modo floating}

Dentre as modalidades eletrocinéticas, o modo floating, também denominado de unpinched, é, do ponto de vista instrumental, o procedimento mais simples para injeção da amostra nos MSE. Isso se justifica pelo fato de o protocolo exigir apenas uma fonte de alta tensão monocanal. ${ }^{76}$ A Figura 2 mostra um esquema do controle eletrocinético para as etapas de injeção e separação em MSE

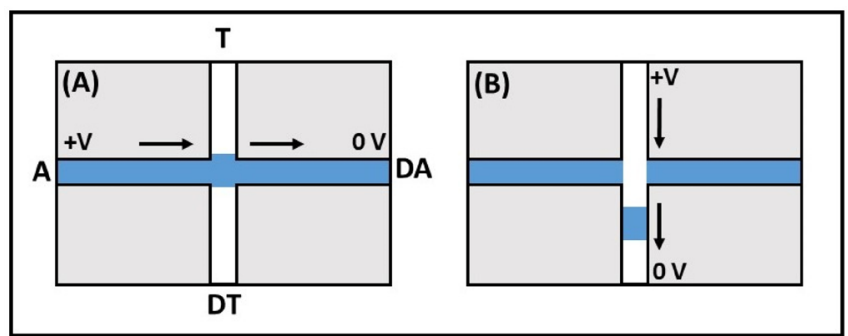

Figura 2. Representação do procedimento utilizado para promover a injeção eletrocinética no modo "floating”. Em (A), observa-se o preenchimento do canal de injeção. Em (B), observa-se a introdução de zona de amostragem, definida na interseção dos canais, no interior do canal de separação. Os indicadores A, T, DA e DT representam os reservatórios da amostra, tampão, descarte da amostra e descarte do tampão, respectivamente. Os potenciais aplicados nas etapas de injeção e separação estão representados, genericamente, por $+V$ 
definidos na configuração de cruz, contendo um canal de injeção (ou amostragem), delimitado pelos reservatórios da amostra (A) e descarte da amostra (DA), e um canal de separação, delimitado pelos reservatórios do tampão (T) e descarte do tampão (DT). Essa geometria é uma das mais utilizadas nos MSE.

Nessa configuração, o controle eletrocinético contemplando as etapas de injeção e separação pode ser explicado em duas etapas. Inicialmente, a solução da amostra é adicionada ao reservatório da amostra e todos os demais reservatórios são preenchidos com solução tampão. Em seguida, uma diferença de potencial elétrico é aplicada entre os reservatórios do canal de injeção possibilitando seu preenchimento com a amostra a ser analisada. Após o preenchimento, o potencial aplicado ao canal de injeção é desligado e um novo potencial é aplicado ao canal de separação. Nessa etapa, o volume definido geometricamente pela intersecção dos canais corresponde à zona de amostra a ser introduzida no interior do canal de separação. ${ }^{79}$ Considerando, como exemplo hipotético, um dispositivo no formato de cruz (Figura 3A) contendo canais definidos com largura e profundidade de $50 \mu \mathrm{m}$, o volume de injeção definido na intersecção seria de 125 pL. É importante destacar que o volume na intersecção dos canais é definido baseando-se na área geométrica e na profundidade do canal. Como a largura de ambos os canais e a profundidade no exemplo citado são iguais, o volume é calculado considerando um cubo com aresta de $50 \mu \mathrm{m}$. Dependendo do sistema de detecção acoplado aos MSE, o volume da ordem de $\mathrm{pL}$ pode ser um problema em função dos níveis de detectabilidade. Para contornar esse problema, uma das estratégias é aumentar o volume de amostra, o que envolve a mudança na configuração dos canais, como ilustrado na Figura 3.

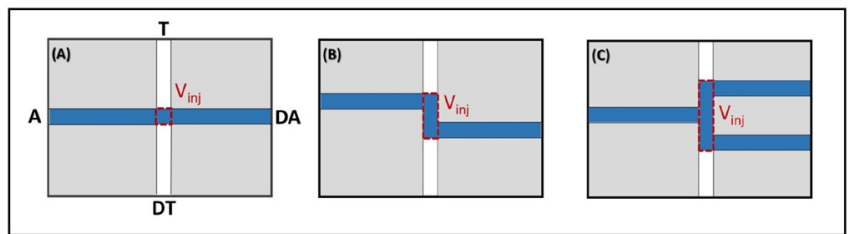

Figura 3. Representação da intersecção dos canais nos arranjos em cruz (A), duplo-T $(B)$ ou triplo-T $(C)$ para definição geométrica do volume de injeção $\left(V_{i n j}\right)$. Outras informações: ver Figura 2

Em geral, a geometria no formato de cruz (Figura 3A) pode ser alterada para os formatos de duplo-T ou triplo-T, como representado nas Figuras 3B e 3C, respectivamente. Nesses arranjos, o volume de amostra depende do comprimento da região definida pela distância entre os canais (gap). Obviamente, quanto maior o gap, maior o volume de amostra. Dessa forma, visando o melhor desempenho analítico, o volume ideal para introdução da amostra precisa ser avaliado tendo em vista o sistema de detecção acoplado aos MSE. $\mathrm{O}$ volume em excesso pode comprometer a eficiência da separação eletroforética. Embora esse modo de injeção apresente simplicidade instrumental, o principal desafio é controlar a difusão da amostra durante a etapa de preenchimento do canal de injeção. A difusão pode promover um aumento no comprimento da zona de amostragem, contribuindo para o alargamento do pico, afetando assim a eficiência de separação e também a reprodutibilidade das injeções. ${ }^{79,80}$

Gabriel e colaboradores reportaram um estudo comparando o efeito da geometria dos canais no volume de amostra introduzido no interior do canal de separação. Os autores avaliaram dispositivos desenvolvidos em PT nas configurações de cruz, simples-T e duplo-T para a separação e detecção de cátions inorgânicos usando um detector condutométrico sem contato. ${ }^{81}$ Para o dispositivo projetado no formato de cruz, o volume definido na intersecção dos canais $(200 \mathrm{pL})$ foi insuficiente para promover variação na resposta analítica do detector referente à mistura catiônica. Para o formato de simples-T, os autores observaram uma separação incompleta devido ao volume excessivo de amostra $(600 \mathrm{pL})$ introduzido no canal de separação. Por fim, o dispositivo fabricado em formato de duplo-T permitiu a formação de uma zona de amostragem como volume suficiente $(400 \mathrm{pL})$ para permitir variações no sinal analítico registrado no detector. Essa configuração assegurou separações eletroforéticas com desempenho analítico satisfatório.

\section{Modo pinched}

O modo de injeção conhecido como pinched se difere do modo floating pelo fato de se aplicar potenciais elétricos em três reservatórios simultaneamente, conforme ilustrado na Figura 4. Em geral, esse modo de injeção envolve duas etapas. Inicialmente, potenciais elétricos representados por $+\mathrm{V}_{1},+\mathrm{V}_{2} \mathrm{e}+\mathrm{V}_{3}$ são aplicados simultaneamente nos reservatórios $\mathrm{A}, \mathrm{T}$ e $\mathrm{DT}$, mantendo o reservatório DA aterrado. O controle eletrocinético simultâneo gera, consequentemente, um confinamento do fluxo da solução de amostra entre a intersecção dos canais e o reservatório DA. ${ }^{79,82} \mathrm{Em}$ seguida, um potencial elétrico é aplicado no canal de separação concomitantemente com a aplicação de dois potenciais auxiliares, representados por $-\mathrm{V}_{3} \mathrm{e}-\mathrm{V}_{4}$, nos reservatórios A e DA (Figura 4C). Enquanto a diferença de potencial aplicada no canal de separação propicia a separação, os potenciais auxiliares têm a função de evitar a dispersão da amostra, permitindo assim a formação de uma zona de amostragem homogênea e simétrica. ${ }^{76,83,84}$

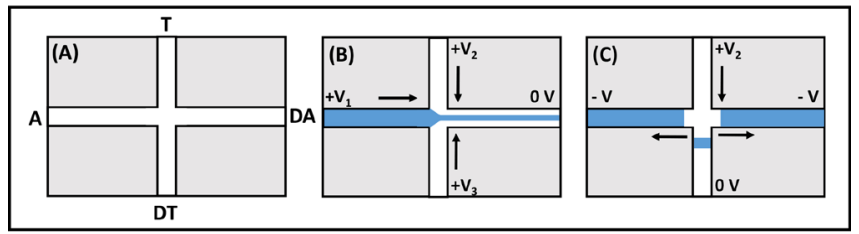

Figura 4. Representação do procedimento utilizado para promover a injeção eletrocinética no modo "pinched" explorando a geometria definida (A) no formato de cruz. Em (B), observa-se o preenchimento do canal de injeção com o confinamento da amostra a partir da intersecção dos canais. Em (C), nota-se a introdução do volume de amostra no interior do canal de separação. Os potenciais aplicados nas etapas de injeção e separação estão representados, genericamente, por $+V_{1},+V_{2} e+V_{3}$

Em comparação ao modo floating, a quantidade de amostra introduzida nos canais de separação depende majoritariamente das forças eletrocinéticas, não tendo influência do tempo de confinamento ou da geometria do canal. ${ }^{16,76,79}$ Alarie e colaboradores realizaram um estudo fundamental avaliando o efeito do campo elétrico aplicado no modo pinched e suas implicações no comprimento da zona de amostra formado. ${ }^{82} \mathrm{Em}$ geral, os autores demonstraram que quanto maior a intensidade do potencial elétrico aplicado em $\mathrm{V}_{2} \mathrm{e} \mathrm{V}_{3}$ (ver Figura 4B), por exemplo, menor o volume de amostra injetado no interior do canal e, consequentemente, maior a eficiência de separação. $\mathrm{O}$ trabalho reportado pelos autores permite uma avaliação das condições electrocinéticas visando maximizar a eficiência de separação e manter um bom equilíbrio com os níveis de detectabilidade.

\section{Modo gated}

O modo de introdução da amostra conhecido como gated se baseia em um protocolo em que o tempo de difusão da amostra define o volume de injeção a ser introduzido no interior do canal de separação. ${ }^{76}$ Nesse processo, se faz necessário a aplicação, concomitantemente, de potenciais elétricos em dois canais microfluídicos. Para preencher o canal de amostragem, aplica-se uma diferença de potencial $\left(+\mathrm{V}_{1}\right)$ no canal definido entre os reservatórios A e DA. Simultaneamente, é estabelecida uma diferença de potencial $\left(+\mathrm{V}_{2}\right)$ no canal definido 
entre os reservatórios T e DT. Nessa etapa, o transporte eletrocinético promove o preenchimento dos canais com as respectivas soluções, conforme visualizado na Figura 5A. Para a formação do plug de amostra, o potencial aplicado no reservatório $\mathrm{T}$ é desligado por um curto intervalo de tempo (tipicamente entre 1 e $5 \mathrm{~s}$ ), permitindo a difusão da amostra para o interior do canal de separação (Figura 5B). Ao reestabelecer o potencial aplicado inicialmente, um volume de amostra definido pelo tempo de difusão é então introduzido no canal de separação (Figura 5C). Em seguida, a separação eletroforética ocorre normalmente e as espécies são usualmente detectadas no final do canal de separação. ${ }^{85-87}$ Nessa metodologia, o volume de amostra introduzido no canal de separação depende diretamente do tempo de injeção e da magnitude do campo elétrico aplicado. ${ }^{14}$

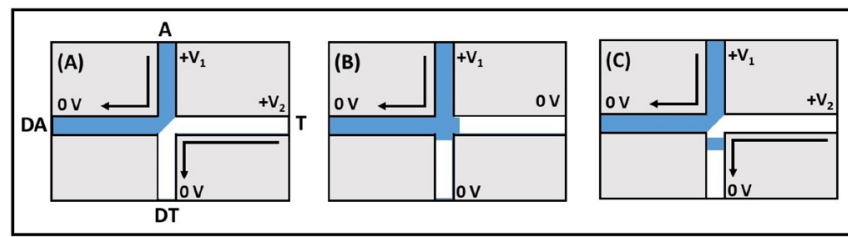

Figura 5. Representação do procedimento utilizado para promover a injeção eletrocinética no modo "gated". Em (A), observa-se o preenchimento dos canais com tampão e amostra. Em (B), observa-se a difusão da amostra ao desligar o potencial $+V_{2}$ aplicado no reservatório $T$. Em $(C)$, quando o potencial $+V_{2}$ é reestabelecido, promove-se a introdução de uma zona de amostra no interior do canal de separação

A injeção da amostra explorando o modo gated pode ser utilizada em associação com diferentes detectores. Jacobson e colaboradores demonstraram, de forma pioneira, o uso da injeção no modo gated para análise de aminoácidos em MSE acoplados com detecção por fluorescência.$^{86}$ Os autores reportaram que o tempo de injeção de $100 \mathrm{~ms}$ foi suficiente para permitir a injeção de volumes entre 60 e $100 \mathrm{pL}$. Uma das principais vantagens do modo gated inclui a possibilidade de realizar o preenchimento dos canais de forma contínua e, consequentemente, permitir o processamento de múltiplas injeções. Freitas e colaboradores descreveram o uso desse modo de injeção em MSE acoplados com um detector condutométrico sem contato para a análise de íons inorgânicos em amostras ambientais bem como para o monitoramento do ciclo do nitrogênio em um ambiente aquático. ${ }^{88} \mathrm{~A}$ estratégia adotada pelos autores permitiu a realização de 30 injeções consecutivas com excelente repetitividade. Huynh e coautores demonstraram o acoplamento do modo gated com a técnica de microdiálise visando a análise de compostos de interesse bioanalítico usando um sistema de detecção por fluorescência. ${ }^{89}$ Uma estratégia similar foi adotada por Mecker e Scott Martin, porém, empregando-se detecção amperométrica para a análise de neurotransmissores. ${ }^{90}$

Com relação aos três modos eletrocinéticos para injeção da amostra, vale ressaltar que vários autores desenvolveram modelos matemáticos e reportaram simulações computacionais para uma melhor compreensão dos fenômenos relacionados à distribuição das linhas do campo elétrico nas diferentes etapas apresentadas. ${ }^{16,91-94}$ Os modelos teóricos desenvolvidos foram comparados com os resultados experimentais e permitem um entendimento conceitual das etapas envolvidas na injeção da amostra.

\section{Injeção discriminatória da amostra}

Embora os métodos eletrocinéticos apresentem simplicidade instrumental, a injeção da amostra através dos modos floating e gated discutidos anteriormente proporciona, indesejavelmente, a discriminação da quantidade de amostra introduzida no interior dos microcanais. ${ }^{79,86,95}$ Isso ocorre devido ao fato da quantidade de amostra ser dependente da magnitude do FEO e também da mobilidade eletroforética. ${ }^{75,96,97}$ De forma simplificada, espécies com maior mobilidade são introduzidas em quantidades superiores às espécies de menor mobilidade. Essa discriminação afeta a reprodutibilidade e a análise quantitativa. ${ }^{79,87,98}$

Dentre os trabalhos reportados na literatura, observa-se que o uso de injetores hidrodinâmicos em MSE minimiza o efeito de discriminação dos analitos na etapa injeção, assim como acontece nos sistemas convencionais de EC. ${ }^{77}$ A comparação entre modos de injeção por pressão e os modos eletrocinéticos demonstra que o uso de injetores hidrodinâmicos favorece a formação de picos mais simétricos e estreitos, além de proporcionar melhor reprodutibilidade e eficiência de separação. ${ }^{96-101}$

Em 2008, Wang e colaboradores propuseram uma estratégia diferente para introdução da amostra baseada na inclinação do MSE com o objetivo de promover a injeção por diferença de pressão. ${ }^{99}$ No experimento realizado, os autores utilizaram uma mistura equimolar contendo dopamina e catecol como modelo, cujos analitos foram separados e detectados eletroquimicamente na saída do canal de separação. Adicionalmente, os autores promoveram a mesma separação, porém, utilizando-se a injeção eletrocinética da amostra. Conforme visualizado na Figura 6, o sinal eletroquímico obtido para dopamina via injeção eletrocinética apresentou-se muito mais intenso em comparação à injeção por pressão. Essa diferença é atribuída à discriminação eletroforética durante a injeção eletrocinética, uma vez que a dopamina apresenta maior mobilidade em relação ao catecol. Dessa forma, uma das estratégias para eliminar ou minimizar o efeito da injeção discriminatória da amostra envolve a implementação dos modos de injeção hidrodinâmica nos MSE, que será discutido no próximo subtópico.
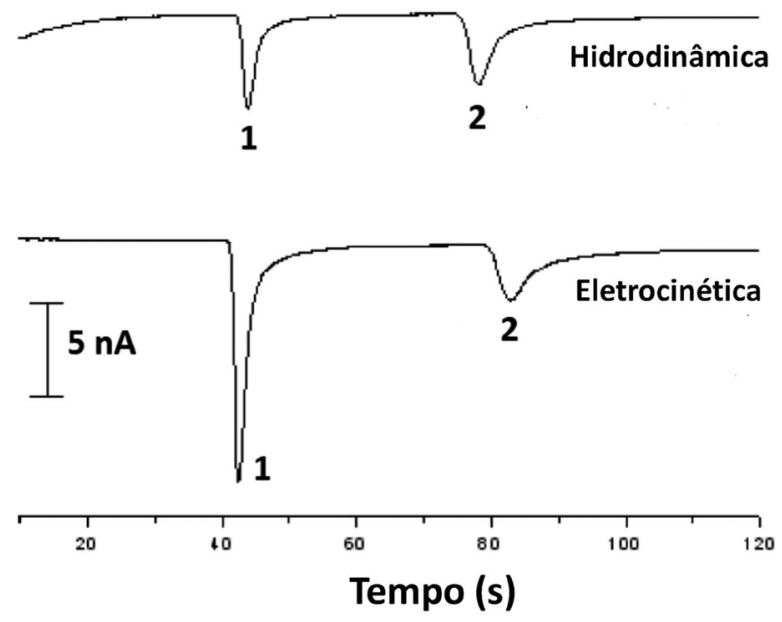

Figura 6. Eletroferogramas comparando o desempenho dos modos hidrodinâmico e eletrocinético para injeção de uma amostra modelo contendo (1) dopamina e (2) catecol (500 $\mu \mathrm{mol} \mathrm{L}^{-1}$ cada). Figura adaptada e reimpressa da referência 99 , com permissão

\section{Injeção hidrodinâmica}

Em comparação à injeção eletrocinética, o controle hidrodinâmico no processo de introdução da amostra nos MSE é menos usual, embora seu uso tenha aumentado consideravelmente nos últimos anos. Isso se deve à necessidade de acoplamento ou interfaceamento com bombas e válvulas ou outros dispositivos similares para esta finalidade. ${ }^{75}$

Em geral, o processo de introdução da amostra no modo hidrodinâmico se dá por meio da gravidade ou uso da pressão. Nos sistemas convencionais de EC, o volume de amostra injetado no interior dos capilares está relacionado ao raio e ao comprimento do capilar, bem como à variação de altura ou pressão e às características 
específicas da amostra, como viscosidade e densidade. ${ }^{102,103}$ As equações 2 e 3 descrevem a dependência do volume de injeção em relação aos parâmetros mencionados:

$$
\begin{gathered}
V_{i}=\frac{\rho g \pi r^{4} t_{i} \Delta h}{8 \eta L} \\
V_{i}=\frac{\pi r^{4} t_{i} \Delta P}{8 \eta L}
\end{gathered}
$$

em que $\rho$ é a densidade da amostra, $g$ é a aceleração da gravidade, $r$ é o raio do capilar, $t_{i}$ é o tempo de injeção, $\Delta h$ é a diferença de altura entre o reservatório de introdução de amostra e de descarte, $\eta$ é a viscosidade da amostra, $L$ é o comprimento do capilar e $\Delta P$ representa a variação de pressão.

Dessa forma, o processo de injeção hidrodinâmica independe das magnitudes das mobilidades eletroforética e eletrosmótica e, consequentemente, não discrimina a quantidade de amostra a ser injetada no interior dos capilares, bem como dos microcanais. Como já mencionado, um dos principais desafios para implementação da injeção hidrodinâmica em MSE se relaciona à conexão dos MSE com os diferentes sistemas de bombeamento, muitos dos quais requerem o controle automatizado além de válvulas e bombas microfabricadas. ${ }^{104,105}$ No entanto, nos últimos anos, sistemas alternativos foram reportados proporcionando simplicidade instrumental para essa finalidade. Nesse contexto, as próximas subseções desta revisão visam apresentar e discutir os principais modos de injeção hidrodinâmica, destacando-se o uso de válvulas e bombas microfabricadas, bombas seringa, micropipeta eletrônica, pipetadores manuais e agulhas de acupuntura.

\section{Injeção hidrodinâmica com bombas peristálticas e válvulas microfabricadas}

O uso das técnicas convencionais de microfabricação, especialmente a fotolitografia, tem viabilizado a construção de sistemas microfluídicos integrados com outros componentes incluindo bombas e válvulas. Bowen e Martin desenvolveram um MSE integrado com três bombas peristálticas e duas válvulas microfabricadas em uma plataforma contendo duas camadas de PDMS (ver Figura 7). ${ }^{104}$ As bombas peristálticas foram utilizadas para
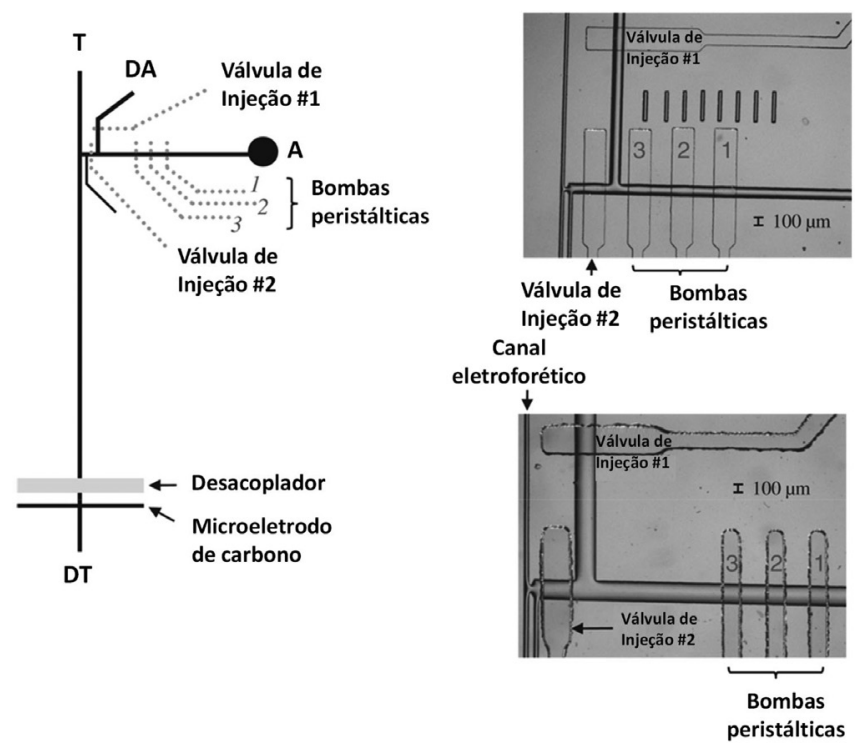

Figura 7. Exemplo de microssistema eletroforético integrado com bombas peristálticas e válvulas microfabricadas. Figura adaptada e reimpressa da referência 104, com permissão bombear a solução da amostra do reservatório até a válvula de injeção. Por sua vez, as válvulas atuaram como interface entre o bombeamento hidrodinâmico e o canal de separação, no qual um potencial elétrico foi aplicado para promover a separação dos analitos. A injeção da amostra foi controlada abrindo-se a válvula 2 por um intervalo de tempo definido. As válvulas do dispositivo foram controladas através do uso de válvulas MAC (MAC Fluid Power Engineering, St. Louis, MO, EUA) que possuem uma unidade controle com temporizador. ${ }^{106}$ Conforme reportado, baseando-se na abertura da válvula 2 durante $7 \mathrm{~s}$, o volume de amostra introduzido no microcanal de separação foi estimado em $670 \mathrm{pL}$. Os autores demonstraram com sucesso a injeção, a separação e a detecção de uma mistura contendo dopamina e norepinefrina usando um MSE fabricado em PDMS contendo um canal de separação com comprimento igual a $3 \mathrm{~cm}$.

Sahore e colaboradores reportaram o desenvolvimento de um MSE integrado com uma bomba peristáltica e válvulas pneumáticas, integralmente fabricado em PDMS. ${ }^{105}$ A viabilidade do dispositivo integrado foi demonstrada através da separação de aminoácidos, peptídeos e proteínas. A injeção hidrodinâmica da amostra, realizada por meio das válvulas pneumáticas, apresentou desempenho superior à injeção eletrocinética, especificamente em relação à intensidade de pico obtida. A diferença observada para a intensidade dos picos se justifica ao fato do volume de amostra no injetor hidrodinâmico $(300 \mathrm{pL})$ ser maior que o volume injetado eletrocineticamente $(60 \mathrm{pL})$.

Cong e colaboradores demonstraram uma estratégia interessante para promover uma etapa de pré-concentração da amostra antes da injeção hidrodinâmica fazendo uso de válvulas microfabricadas via litografia suave (Figura 8A). Conforme visualizado na Figura 8B, a aplicação de um potencial elétrico com a manutenção da válvula fechada induziu à pré-concentração da amostra na região próxima à interface com o canal de separação. A abertura da válvula (membrana) foi então utilizada para permitir a introdução hidrodinâmica de um pequeno volume de amostra no interior do canal de separação (Figuras 8C e 8D). ${ }^{107}$

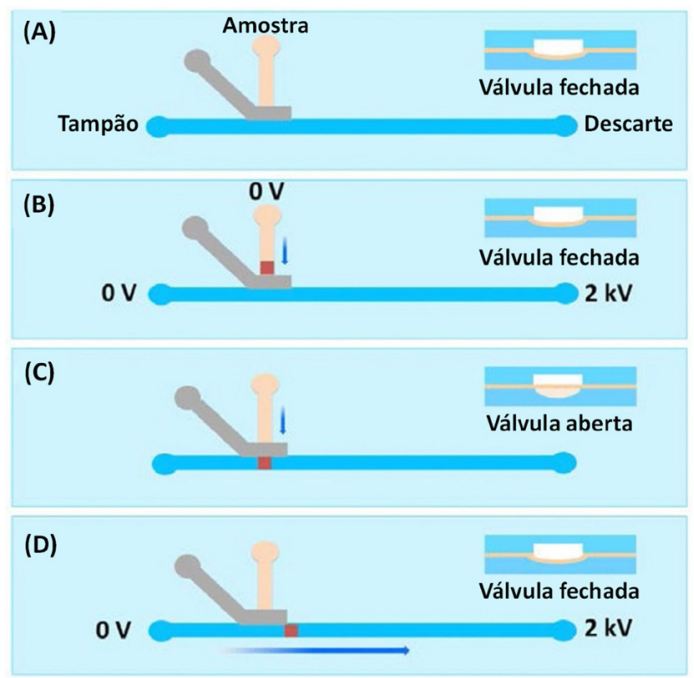

Figura 8. Demonstração do acoplamento de válvulas microfabricadas aos microssistemas eletroforéticos para permitir a pré-concentração eletrocinética da amostra antes da etapa de injeção hidrodinâmica. Figura adaptada e reimpressa da referência 107, com permissão

O uso de válvulas e bombas microfabricadas também foi explorado por outros autores. Detalhes adicionais sobre os demais estudos reportados na literatura podem ser encontrados em artigos de revisão específicos sobre esta modalidade de injeção da amostra em MSE. ${ }^{16,75}$ 
Injeção hidrodinâmica com bombas seringas e micropipeta eletrônica

Tendo em vista a dificuldade instrumental e a complexidade do processo de integração de válvulas e bombas microfabricadas, alguns autores reportaram métodos alternativos para promover a introdução da amostra via bombeamento hidrodinâmico. Esses métodos fazem uso de instrumentos comuns como bombas seringas e micropipetas eletrônicas.

Dossi e colaboradores reportaram o acoplamento de uma bomba seringa a um MSE fabricado em vidro para promover a injeção hidrodinâmica da amostra (Figura 9A). ${ }^{100}$ A bomba seringa foi conectada ao MSE por meio do uso de um tubo de aço inoxidável integrado ao reservatório da amostra. A amostra foi introduzida no interior do canal de injeção através do controle da vazão do fluxo induzido pela bomba seringa. De acordo com os resultados publicados, os melhores resultados foram obtidos aplicando-se uma vazão de $8 \mu \mathrm{L} \mathrm{min}{ }^{-1}$ durante $6 \mathrm{~s}$. Em seguida, o campo elétrico foi aplicado entre os reservatórios do tampão e descarte do tampão permitindo a introdução do plug de amostra no interior do canal de separação. Simultaneamente à etapa de separação, um potencial elétrico também foi aplicado aos reservatórios da amostra e do descarte da amostra para evitar introdução indesejada da amostra causadas pela dispersão. A viabilidade do método proposto foi demonstrada através da injeção de uma amostra modelo contendo catecol empregando-se a detecção amperométrica na saída no canal de separação. Os autores realizaram a comparação da injeção hidrodinâmica proposta com a injeção eletrocinética. Conforme apresentado na Figura 9B, o desempenho analítico da injeção hidrodinâmica por meio da bomba seringa apresentou melhor repetitividade $(n=5)$ e proporcionou melhor eficiência de separação quando comparado à injeção eletrocinética.

Gabriel e colaboradores demonstraram o uso de uma micropipeta eletrônica para promover a injeção controlada da amostra nos MSE. ${ }^{97}$ A micropipeta foi acoplada a um MSE fabricado em material híbrido composto de PDMS/vidro. Basicamente, os microcanais foram definidos em PDMS a partir de um processo de litografia suave usando moldes alternativos ${ }^{61}$ e selados contra uma membrana de PDMS parcialmente curada e depositada sobre uma base de vidro. Para facilitar a conexão da micropipeta com o dispositivo, tubos de silicone foram integrados ao PDMS durante a etapa de moldagem. Além disso, conforme visualizado na Figura 9C, um canal adicional foi integrado paralelamente ao canal de separação para promover a divisão da amostra e assegurar reprodutibilidade no processo de injeção. A prova de conceito do injetor alternativo foi avaliada

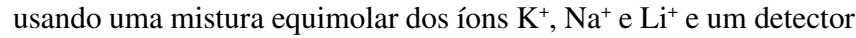
de condutividade sem contato. $\mathrm{O}$ uso da micropipeta eletrônica como injetor foi comparado com o modo de injeção floating e, além de apresentar um perfil de injeção sem discriminação da amostra, ofereceu melhor desempenho analítico em comparação à injeção eletrocinética. Como pode ser visualizado na Figura 9D, as áreas dos picos obtidos via injeção hidrodinâmica, ao contrário da injeção eletrocinética, não apresentaram variação significativa quando a mistura modelo foi analisada em um sistema tampão preparado em diferentes concentrações.

\section{Injeção hidrodinâmica com pipetadores manuais e agulhas de acupuntura}

Além dos exemplos citados na seção anterior, outras propostas alternativas de injetores hidrodinâmicos foram descritas na literatura,
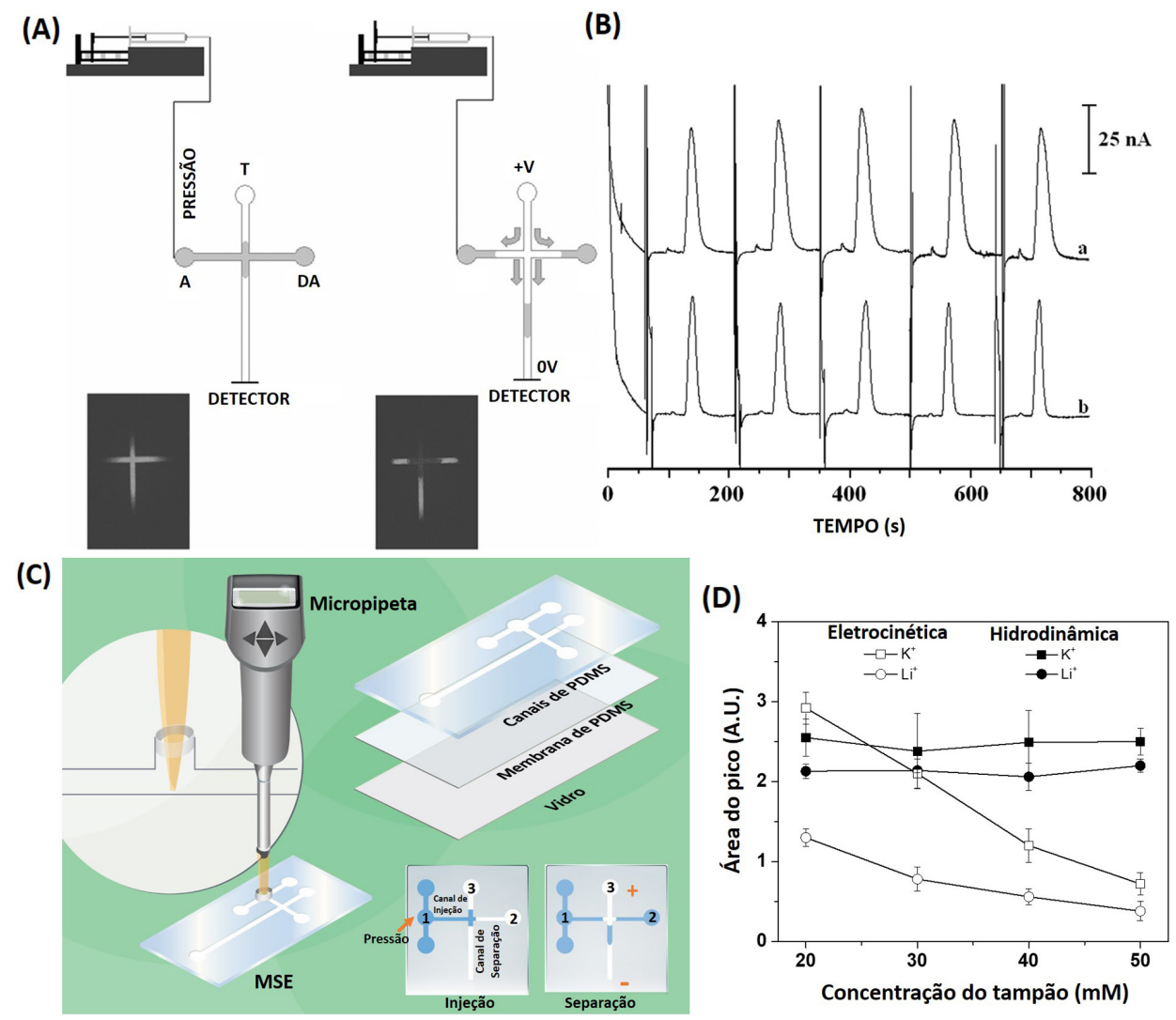

Figura 9. Representação de injetores hidrodinâmicos alternativos para MSE incluindo o uso de bombas seringas e micropipetas eletrônicas. (A) Acoplamento de bomba seringa a um MSE. (B) Eletroferogramas mostrando a análise de catecol através da injeção hidrodinâmica e injeção eletrocinética. (C) Acoplamento de uma micropipeta eletrônica a um canal auxiliar em um MSE híbrido composto de PDMS/vidro. (D) Variação da área de pico em função da concentração do tampão utilizado no procedimento eletroforético adotado durante a injeção hidrodinâmica e injeção eletrocinética. Figuras adaptadas e reimpressas das referências 97 e 100, com permissão 


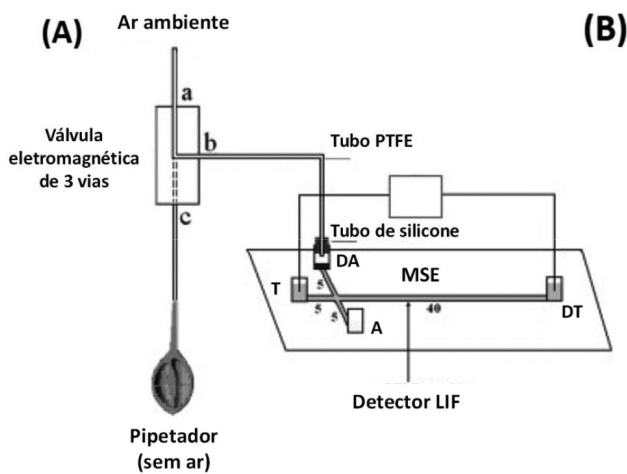

(B)
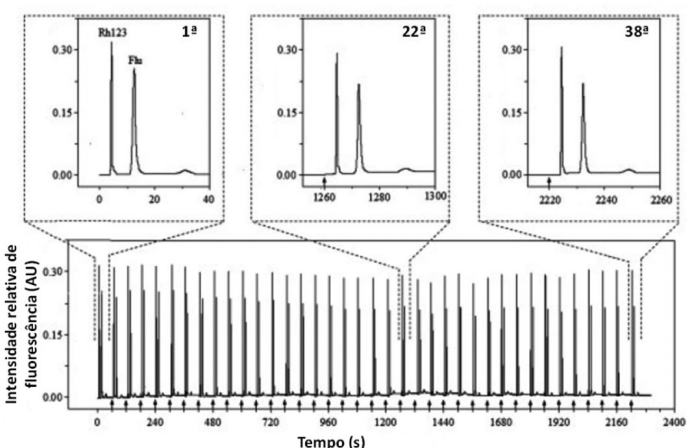

Figura 10. Representação do uso de um pipetador manual conectado a uma válvula eletromagnética de 3 vias como injetor para MSE e eletroferogramas resultantes mostrando uma série de 38 injeções sequenciais de uma amostra modelo contendo rodamina e fluoresceína. Figura adaptada e reimpressa da referência 108, com permissão

como o uso de um pipetador (pera) e uma válvula eletromagnética de 3 vias $^{108}$ ou uma agulha de acupuntura. ${ }^{101} \mathrm{Hu}$ e colaboradores reportaram o uso de um pipetador para injeção hidrodinâmica por pressão negativa (Figura 10). Os autores utilizaram um MSE de vidro, fabricado por processo fotolitográfico, com canais de 20 $\mu \mathrm{m}$ de profundidade e $60 \mu \mathrm{m}$ de largura. $\mathrm{O}$ dispositivo utilizado foi baseado na geometria de uma cruz e seus reservatórios foram confeccionados com a base de ponteiras de micropipetas de modo a apresentarem um volume de aproximadamente $200 \mu \mathrm{L}$. A amostra a ser introduzida nos microcanais foi colocada no reservatório A (Figura 10A) enquanto o reservatório DA foi conectado a uma das saídas da válvula eletromagnética de três vias. A injeção da amostra foi promovida manualmente ao pressionar o pipetador, conectado a outra extremidade da válvula. Os autores demonstraram a viabilidade do injetor proposto através da separação de rodamina e fluoresceína utilizando-se um detector de fluorescência induzida a laser. O injetor proposto ofereceu ótima repetitividade (Figura 10B), com desvios padrões relativos entre 1,3 e $2,2 \%$ para um total de trinta de oito injeções sequenciais. ${ }^{108}$

Ha e Hahn propuseram o uso de uma agulha de acupuntura como injetor hidrodinâmico para MSE poliméricos. ${ }^{101}$ Com o auxílio de uma seringa, a agulha foi preenchida com a amostra e introduzida verticalmente em um MSE, perfurando a parte polimérica superior do dispositivo (Figura 11A). A solução da amostra foi introduzida no canal aplicando-se uma pressão externa ao acoplar uma seringa com um micrômetro na agulha. A capacidade volumétrica da seringa, juntamente com o uso do micrômetro, possibilitou injeções de aproximadamente $3 \mathrm{~nL}$ de amostra. O desempenho do injetor hidrodinâmico proposto foi comparado com os modos eletrocinéticos conhecidos como gated e pinched usando-se uma mistura modelo contendo fluoresceína e isotiocianato de fluoresceína. (Figura 11B). Como pode ser visualizado nos eletroferogramas apresentados na Figura 11B, não foram observadas diferenças significativas entre os modos de injeção avaliados. Em contrapartida, o injetor proposto apresentou a vantagem de permitir injeções seriadas da amostra em qualquer posição do MSE.

\section{CONCLUSÕES E PERSPECTIVAS}

Este estudo apresentou, de forma inédita, uma revisão em língua portuguesa sobre os métodos de introdução da amostra em microssistemas eletroforéticos, abordando aspectos teóricos e instrumentais relacionados aos modos eletrocinéticos e hidrodinâmicos. Dentre os modos eletrocinéticos, foram abordadas estratégias para o aumento do volume de amostra a ser introduzido nos microcanais bem como três protocolos para o controle fluídico durante o procedimento de injeção. Com relação à injeção hidrodinâmica, métodos sofisticados
(A)

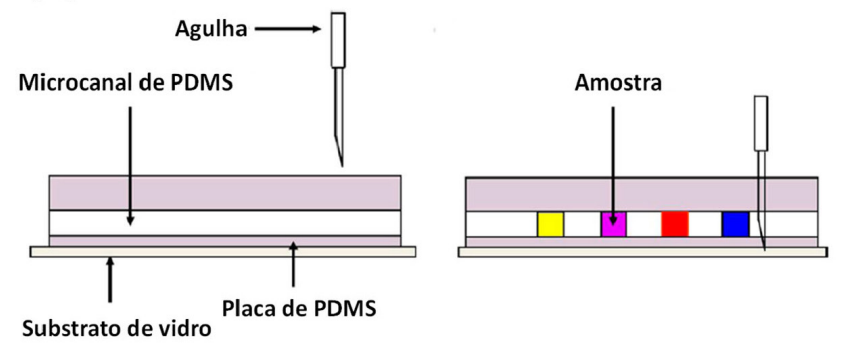

(B) Modo de injeção gated Modo de injeção pinched
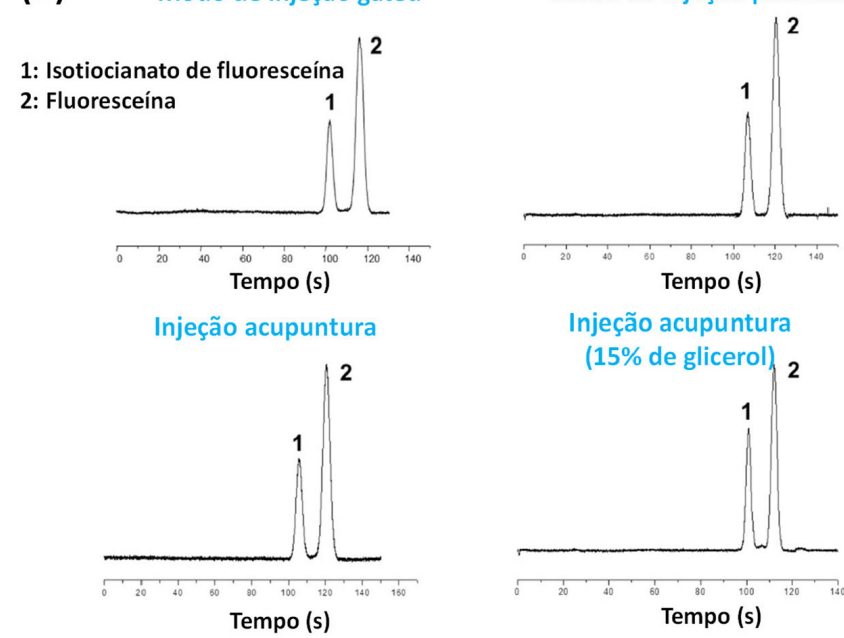

Figura 11. (A) Injetor hidrodinâmico para MSE baseado no uso de uma agulha de acupuntura e (B) eletroferogramas mostrando a separação de uma mistura contendo fluoresceína e isotiocianato de fluoresceína introduzida no interior dos microcanais com a agulha e por meio dos modos eletrocinéticos conhecidos como "gated" $e$ "pinched". Figura adaptada e reimpressa da referência 101, com permissão

contendo válvulas e bombas microfabricadas devidamente integradas aos microssistemas foram devidamente apresentados e discutidos. Além disso, o desenvolvimento de estratégias alternativas incluindo o uso de bombas seringas, pipetas eletrônicas, pipetadores manuais e agulhas de acupuntura também foi abordado, evidenciando a simplicidade instrumental e demonstrando que métodos simples podem ser implementados de forma independe das tecnologias de alto custo ou de ambientes específicos como salas limpas.

Em virtude do aumento crescente do uso de sistemas miniaturizados em aplicações envolvendo separações eletroforéticas, o conhecimento dos fundamentos, das limitações e dos diferentes procedimentos 
operacionais certamente pode impulsionar o desenvolvimento científico na área relacionada à microfluídica. Dessa forma, a revisão apresentada preenche uma lacuna existente na literatura nacional que pode, indubitavelmente, contribuir com a formação de jovens pesquisadores ou iniciantes na área da eletroforese em microssistemas.

\section{AGRADECIMENTOS}

Os autores agradecem ao CNPq (processos 426496/2018-3 e 308140/2016-8), à CAPES, à FAPEG e ao INCTBio (processo 465389/2014-7) pelo apoio financeiro e bolsas concedidas.

\section{REFERÊNCIAS}

1. Evenhuis, C. J.; Guijt, R. M.; Macka, M.; Haddad, P. R.; Electrophoresis 2004, 25, 3602.

2. Jokerst, J. C.; Emory, J. M.; Henry, C. S.; Electrophoresis 2012, 137, 24.

3. Kovarik, M. L.; Ornoff, D. M.; Melvin, A. T.; Dobes, N. C.; Wang, Y.; Dickinson, A. J.; Gach, P. C.; Shah, P. K.; Allbritton, N. L.; Anal. Chem. 2013, 85, 451 .

4. Castro, E. R.; Manz, A.; J. Chromatogr. A 2015, 1382, 66.

5. Regis, T.; Cesar, L.; Oliveira, M.; Anal. Methods 2018, 10, 5135.

6. Coltro, W. K. T.; Piccin, E.; Carrilho, E.; De Jesus, D. P.; Fracassi Da Silva, J. A.; Da Silva, H. D. T.; Do Lago, C. L.; Quim. Nova 2007, 30, 1986.

7. Reyes, D. R.; Iossifidis, D.; Auroux, P. A.; Manz, A.; Anal. Chem. 2002, 74, 2623.

8. Auroux, P. A.; Iossifidis, D.; Reyes, D. R.; Manz, A.; Anal. Chem. 2002, $74,2637$.

9. Vilkner Janasek, D., Manz, A., T.; Anal. Chem. 2004, 76, 3373.

10. Dittrich, P. S.; Tachikawa, K.; Manz, A.; Anal. Chem. 2006, 78, 3887.

11. West, J.; Becker, M.; Tombrink, S.; Manz, A.; Anal. Chem. 2008, 80, 4403.

12 Ohno, K. I.; Tachikawa, K.; Manz, A.; Electrophoresis 2008, 29, 4443.

13. Arora, A.; Simone, G.; Salieb-Beugelaar, G. B.; Kim, J. T.; Manz, A.; Anal. Chem. 2010, 82, 4830.

14. Roddy, E. S.; Xu, H.; Ewing, A. G.; Electrophoresis 2004, 25, 229.

15. Lacher, N. A.; Garrison, K. E.; Martin, R. S.; Lunte, S. M.; Electrophoresis 2001, 22, 2526.

16. Karlinsey, J. M.; Anal. Chim. Acta 2012, 725, 1.

17. Maguire, I.; O'Kennedy, R.; Ducrée, J.; Regan, F.; Anal. Methods 2018, 10, 1497.

18. Kar, S.; Das, S. S.; Laha, S.; Chakraborty, S.; Ind. Eng. Chem. Res. 2020, 59, 3644.

19. Whitesides, G. M.; Nature 2006, 442, 368.

20. Lo, R.; Chem. Eng. Process Tech. 2013, 1, 1.

21. Brum, J. A.; Meneghini, R.; São Paulo em Perspectiva 2002, 16, 48.

22. Wang, J.; Talanta 2002, 56, 223.

23. Horsman, K. M.; Ph, D.; Bienvenue, J. M.; Ph, D.; Blasier, K. R.; Landers, J. P.; J. Forensic Sci. 2007, 52, 784.

24. Sassa, F.; Morimoto, K.; Satoh, W.; Suzuki, H.; Electrophoresis 2008, 29, 1787.

25. Kim, Y. T.; Heo, H. Y.; Lee, S. H.; Kim, D. H.; Seo, T. S.; Electrophoresis 2015, 36, 1728.

26. Spikmans, V.; WIREs Forensic Sci. 2019, $1,1$.

27. Ferey, L.; Delaunay, N.; Sep. Purif. Rev. 2016, 45, 193.

28. Saylor, R. A.; Lunte, S. M.; J. Chromatogr. A 2015, 1382, 48.

29. Pagaduan, J. V; Sahore, V.; Woolley, A. T.; Anal. Bioanal. Chem. 2015, 407, 6911

30. Křřžek, T.; Kubíčková, A.; Anal. Bioanal. Chem. 2012, 403, 2185.

31. Tetala, K. K. R.; Vijayalakshmi, M. A.; Anal. Chim. Acta 2016, 906, 7.

32. Liu, Y.; Wang, W.; Jia, M.; Liu, R.; Liu, Q.; Xiao, H.; Li, J.; Xue, Y.; Wang, Y.; Yan, C.; Electrophoresis 2018, 39, 8.
33. Hosic, S.; Murthy, S. K.; Koppes, A. N.; Anal. Chem. 2016, 88, 354. 34. Kim, J.; Johnson, M.; Hill, P.; Gale, B. K.; Integr. Biol. 2009, 1, 574. 35. Mach, A. J.; Adeyiga, O. B.; Di Carlo, D.; Lab Chip 2013, 13, 1011. 36. Sonker, M.; Sahore, V.; Woolley, A. T.; Anal. Chim. Acta 2017, 986, 1. 37. Melchert, W. R.; Reis, B. F.; Rocha, F. R. P.; Anal. Chim. Acta 2012, 714,8 .

38. Trojanowicz, M.; Kołacińska, K.; Analyst 2016, 141, 2085.

39. Lagus, T. P.; Edd, J. F.; J. Phys. D. Appl. Phys. 2013, 46, 114005.

40. Pfeiffer, S. A.; Nagl, S.; Methods Appl. Fluoresc. 2015, 3, 34003.

41. Reverté, L.; Prieto-Simón, B.; Campàs, M.; Anal. Chim. Acta 2016, 908, 8.

42. Blair, E. O.; Corrigan, D. K.; Biosens. Bioelectron. 2019, 134, 57.

43. Liu, W. wen; Zhu, Y.; Anal. Chim. Acta 2020, 1113, 66.

44. Tavares, M. F. G.; Quim. Nova 1996, 19, 173.

45. Silva, J. A. F. da; Coltro, W. K. T.; Carrilho, E.; Tavares, M. F. M.; Quim. Nova 2007, 30, 740.

46. Simas Vaz, F.; Marta De Oliveira, C.; Leal De Oliveira, M.; Quim. Nova 2015, 38, 732.

47. Breadmore, M. C.; J. Chromatogr. A 2012, 1221, 42.

48. García, C. D.; Liu, Y.; Anderson, P.; Henry, C. S.; Lab Chip 2003, 3, 324.

49. Blanes, L.; Coltro, W. K. T.; Saito, R. M.; Van Gramberg, A.; Lucio do Lago, C.; Doble, P.; Electrophoresis 2012, 33, 893.

50. Zhang, M.; Phung, S. C.; Smejkal, P.; Guijt, R. M.; Breadmore, M. C.; Trends Environ. Anal. Chem. 2018, 18, 1.

51. Caruso, G.; Fresta, C. G.; Siegel, J. M.; Wijesinghe, M. B.; Lunte, S. M.; Anal. Bioanal. Chem. 2017, 409, 4529.

52. Hall, G. H.; Glerum, D. M.; Backhouse, C. J.; Electrophoresis 2016, 37 , 406.

53. Santos, M. R.; Tavares, M. F. M.; Rubim, J. C.; Quim. Nova 2000, 23, 585

54. Nielsen, J. B.; Nielsen, A. V.; Carson, R. H.; Lin, H. J. L.; Hanson, R. L.; Sonker, M.; Mortensen, D. N.; Price, J. C.; Woolley, A. T.; Electrophoresis 2019, 40, 2853.

55. Kitagawa, F.; Otsuka, K.; J. Pharm. Biomed. Anal. 2011, 55, 668.

56. Assunção, N. A.; Bechara, E. J. H.; Simionato, A. V. C.; Tavares, M. F. M.; Carrilho, E.; Quim. Nova 2008, 31, 2124.

57. Matysik, F. M.; Microchim. Acta 2008, 160, 1

58. Della Pelle, F.; Del Carlo, M.; Sergi, M.; Compagnone, D.; Escarpa, A.; Microchim. Acta 2016, 183, 3143.

59. Pinheiro, K. M. P.; Moreira, R. C.; Rezende, K. C. A.; Talhavini, M.; Logrado, L. P. L.; Baio, J. A. F.; Lanza, M. R. V.; Coltro, W. K. T.; Electrophoresis 2019, 40, 2891.

60. Fracassi Da Silva, J. A.; Quim. Nova 2003, 26, 56.

61. Lobo-Júnior, E. O.; Gabriel, E. F. M.; dos Santos, R. A.; de Souza, F. R.; Lopes, W. D.; Lima, R. S.; Gobbi, A. L.; Coltro, W. K. T.; Electrophoresis 2017, 38, 250.

62. Gunawardhana, S. M.; Lunte, S. M.; Anal. Methods 2018, 10, 3737.

63. Piccin, E.; Coltro, W. K. T.; Fracassi da Silva, J. A.; Neto, S. C.; Mazo, L. H.; Carrilho, E.; J. Chromatogr. A 2007, 1173, 151.

64. De Campos, R. P. S.; Campos, C. D. M.; Almeida, G. B.; Fracassi Da Silva, J. A.; IEEE Trans. Biomed. Circuits Syst. 2017, 11, 1470.

65. Gabriel, E. F. M.; Coltro, W. K. T.; Garcia, C. D.; Electrophoresis 2014, $35,2325$.

66. Huang, Z.; Yang, M.; You, H.; Xie, Y.; Electrophoresis 2018, 39, 1802. 67. Shadpour, H.; Hupert, M. L.; Patterson, D.; Liu, C.; Galloway, M.; Stryjewski, W.; Goettert, J.; Soper, S. A.; Anal. Chem. 2007, 79, 870.

68. Wei, X.; Sun, P.; Yang, S.; Zhao, L.; Wu, J.; Li, F.; Pu, Q.; J. Chromatogr. A 2016, 1457, 144.

69. Guo, Y.; Uchiyama, K.; Nakagama, T.; Shimosaka, T.; Hobo, T.; Electrophoresis 2005, 26, 1843.

70. do Lago, C. L.; da Silva, H. D. T.; Neves, C. A.; Brito-Neto, J. G. A.; Fracassi da Silva, J. A.; Anal. Chem. 2003, 75, 3853.

71. Gabriel, E. F. M.; Lucca, B. G.; Duarte, G. R. M.; Coltro, W. K. T.; Anal. Methods 2018, 10, 2952. 
72. Chagas, C. L. S.; De Souza, F. R.; Cardoso, T. M. G.; Moreira, R. C.; Da Silva, J. A. F.; De Jesus, D. P.; Coltro, W. K. T.; Anal. Methods 2016, 8 , 6682.

73. Nanthasurasak, P.; Cabot, J. M.; See, H. H.; Guijt, R. M.; Breadmore, M. C.; Anal. Chim. Acta 2017, 985, 7.

74. Quero, R. F.; Bressan, L. P.; da Silva, J. A. F.; de Jesus, D. P.; Sensors Actuators, B 2019, 286, 301.

75. Saito, R. M.; Coltro, W. K. T.; De Jesus, D. P.; Electrophoresis 2012, 33, 2614.

76. Blas, M.; Delaunay, N.; Rocca, J. L.; Electrophoresis 2008, 29, 20.

77. Baker, D. R.; Capillary Electrophoresis; John Wiley \& Sons: New York, 1995.

78. Wenclawiak, B. W.; Püschl, R. J.; Anal. Lett. 2006, 39, 3.

79. Jacobson, S. C.; Hergenroder, R.; Koutny, L. B.; Warmack, / R J; Ramsey, J. M.; Anal. Chem. 1994, 66, 1107.

80. Shultz-Lockyear, L. L.; Colyer, C. L.; Hugh Fan, Z.; Roy, K. I.; Harrison, D. J.; Electrophoresis 1999, 20, 529.

81. Gabriel, E. F. M.; Duarte Junior, G. F.; Garcia, P. de T.; De Jesus, D. P.; Coltro, W. K. T.; Electrophoresis 2012, 33, 2660.

82. Alarie, J. P.; Jacobson, S. C.; Culbertson, C. T.; Ramsey, J. M.; Electrophoresis 2000, 21, 100.

83. Zhou, W.; Xia, L.; Xiao, X.; Li, G.; Pu, Q.; Electrophoresis 2019, 40, 2165.

84. Alarie, J. P.; Jacobson, S. C.; Michael Ramsey, J.; Electrophoresis 2001, $22,312$.

85. Li, L.; Li, Q.; Chen, P.; Li, Z.; Chen, Z.; Tang, B.; Anal. Chem. 2016, 88,930 .

86. Jacobson, S. C.; Koutny, L. B.; Hergenroder, R.; Moore, A. W.; Ramsey, J. M.; Anal. Chem. 1994, 6664, 3472.

87. Kovarik, M. L.; Lai, H. H.; Xiong, J. C.; Allbritton, N. L.; Electrophoresis 2011, 32, 3180.
88. Freitas, C. B.; Moreira, R. C.; Tavares, M. G. O.; Coltro, W. K. T.; Talanta 2016, 147, 335.

89. Huynh, B. H.; Fogarty, B. A.; Martin, R. S.; Lunte, S. M.; Anal. Chem. 2004, 76, 6440 .

90. Mecker, L. C.; Martin, R. S.; Anal. Chem. 2008, 80, 9257.

91. Fu, L.; Yang, R.; Lee, G.; Liu, H.; Anal. Chem. 2002, 74, 5084.

92. Wu, C. H.; Yang, R. J.; Electrophoresis 2006, 27, 4970.

93. Ermakov, S. V.; Jacobson, S. C.; Ramsey, J. M.; Anal. Chem. 2000, 72, 3512 .

94. Tsai, C. H.; Yang, R. J.; Tai, C. H.; Fu, L. M.; Electrophoresis 2005, 26, 674.

95. Jacobson, S. C.; Ramsey, J. M.; Electrophoresis 1995, 16, 481.

96. Slentz, B. E.; Penner, N. A.; Regnier, F.; Anal. Chem. 2002, 74, 4835.

97. Gabriel, E. F. M.; dos Santos, R. A.; Lobo-Júnior, E. O.; Rezende, K. C. A.; Coltro, W. K. T.; Talanta 2017, 162, 19.

98. Lacharme, F.; Gijs, M. A. M.; Electrophoresis 2006, 27, 2924.

99. Wang, W.; Zhou, F.; Zhao, L.; Zhang, J.; Zhu, J.; Electrophoresis 2008, $29,561$.

100. Dossi, N.; Toniolo, R.; Susmel, S.; Pizzariello, A.; Bontempelli, G.; Electrophoresis 2010, 31, 2541.

101. Ha, J. W.; Hahn, J. H.; Anal. Chem. 2016, 88, 4629.

102. Opekar P.; Tůma, P.; J. Chromatogr. A 2017, 1480, 93.

103. Seiman, A.; Kaljurand, M.; Kubá, P.; J. Chromatogr. A 2011, 1218, 1273.

104. Bowen, A. L.; Martin, R. S.; Electrophoresis 2010, 31, 2534.

105. Sahore, V.; Kumar, S.; Rogers, C. I.; Jensen, J. K.; Sonker, M.; Woolley, A. T.; Anal. Bioanal. Chem. 2016, 408, 599.

106. Li, M. W.; Martin, R. S.; Electrophoresis 2007, 28, 2478.

107. Cong, Y.; Katipamula, S.; Geng, T.; Prost, S. A.; Tang, K.; Kelly, R. T.; Electrophoresis 2016, 37, 455.

108. Hu, H.; Yin, X.; Qi, L.; Liu, J.; Electrophoresis 2009, 30, 4213. 